\title{
Oxidative Imbalance in HIV-1 Infected Patients Treated with Antiretroviral Therapy
}

\author{
Antonella Mandas, ${ }^{1}$ Eugenio Luigi Iorio, ${ }^{2}$ Maria Gabriella Congiu, ${ }^{1}$ Cinzia Balestrieri, ${ }^{1}$ \\ Antonello Mereu, ${ }^{1}$ Daniela Cau, ${ }^{1}$ Sandra Dessì, ${ }^{1}$ and Nicoletta Curreli ${ }^{3}$ \\ ${ }^{1}$ Department of Internal Medical Sciences, University of Cagliari, SS 554 Km 4.5, 09042 Monserrato, Italy \\ ${ }^{2}$ International Observatory of Oxidative Stress, Free Radicals and Antioxidant Systems, Via Paolo Grisignano 21, 84127 Salerno, Italy \\ ${ }^{3}$ Department of Biomedical Science and Technology, Division of Biochemistry, University of Cagliari, SS $554 \mathrm{Km} \mathrm{4.5,}$ \\ 09042 Monserrato, Italy \\ Correspondence should be addressed to Sandra Dessì, sdessi@unica.it
}

Received 10 February 2009; Revised 19 July 2009; Accepted 23 September 2009

Recommended by George Perry

\begin{abstract}
It is generally accepted that oxidative stress is involved in HIV infection. However, the role in oxidative balance of Highly Active Antiretroviral Therapy (HAART) is still debated. In our study we assessed serum oxidant and antioxidant levels in an HIV1 -infected population treated with HAART, and compared them with those of untreated HIV-1 patients and HIV-1-negative subjects. The study included 116 HIV-1-infected patients (86 HAART-treated and 30 untreated), and 46 HIV-negative controls. Serum oxidant levels were significantly higher in the HIV-1 treated group as compared to untreated and control groups. In addition, a decrease of serum total antioxidant status was observed in the HIV-1 treated group. To be noted is that patients who rigorously follow antiretroviral therapy (optimal HAART adherence) have significantly higher oxidative status than those who do not closely follow the therapy (poor HAART adherence). Analysis of variance revealed no significant further increase in oxidative status in HIV-1-infected patients taking antiretroviral and other drugs with the exception of psychiatric drugs (e.g. anxiolytics or antidepressants). Taken together, our results indicate that HAART may affect oxidative stress in HIV-1-infected patients and suggest that antiretroviral therapy plays an important role in the synergy of HIV infection and oxidative stress.
\end{abstract}

Copyright (C) 2009 Antonella Mandas et al. This is an open access article distributed under the Creative Commons Attribution License, which permits unrestricted use, distribution, and reproduction in any medium, provided the original work is properly cited.

\section{Background}

Highly Active Antiretroviral Therapy (HAART) is currently the therapy of choice for HIV-infected patients $[1,2]$. However, despite remarkable viral replication suppression and immune response restoration, long-term HAART appears of limited use in many patients [3], because of additional adverse effects $[4,5]$ and/or regimen adherence difficulties [6]. Furthermore, poor adherence to HAART can have serious consequences, including loss of serum HIV suppression, development of drug-resistant HIV strains, and increased probability of illness progression [6]. It is widely reported that oxidative stress - an imbalance between production and elimination of chemically reactive species, such as Reactive Oxygen Species (ROS) [7]—is involved in HIV infection [8]. Viral Tat protein induces enhanced ROS production in HIV-infected patients by mitochondrial generation of superoxide anion [9], which in turn may activate Nuclear Factor $\kappa \mathrm{B}(\mathrm{NF}-\kappa \mathrm{B})[8]$, thus increasing HIV transcription. It has also been found that HIV infection is able to indirectly inhibit glutathione synthesis [10], which is the main endogen antioxidant.

HAART may increase chemically reactive species in circulation, possibly by producing more oxidized metabolites deriving from the interaction between ROS and infectedcell biomolecules [11-16]. This is supported by several biochemical mechanisms, such as mitochondrial interference, following treatment with HAART-NRTI (Nucleoside Reverse Transcriptase Inhibitors) [14-16], and activation of the P450 hepatic system by HAART, when comprising Protease Inhibitors (PI) [13]. Insufficient intake or malabsorption of nutrients-common in HIV patients-may further worsen 
these conditions $[17,18]$. It has been suggested that immune system reactivation may, itself, be a direct cause of oxidative stress [11] in HAART, when comprising Nonnucleoside Reverse Transcriptase Inhibitors (NNRTI), such as efavirenz. Increased levels of serum isoprostane-a class of bioactive molecules derived from in vivo nonenzymatic oxidation of arachidonic acid-usually observed in smokers [19, 20], were also seen in patients taking efavirenz. On the basis of these findings, we aimed to assess the effect of HAART on oxidative stress in an HIV-1-infected population. To do this we used commercially available tests: (i) d-ROMs test to assess serum oxidative status and (ii) OXY-Adsorbent test to assess serum total antioxidant status.

\section{Methods}

2.1. Subjects. One hundred sixty-two subjects were enrolled by the Department of Internal Medical Sciences, University of Cagliari, Italy. Following written informed consent, they were divided into three groups: (1) HIV-1-negative subjects (controls), (2) HAART-treated HIV-1-infected patients and (3) untreated HIV-1-infected patients, as shown in Table 1. Controls comprised 46 subjects, apparently healthy, on adequate diets, mean age $39.5 \pm 11.4$, of whom 21 females. No control took drugs or nutraceuticals. The second group comprised 86 HIV-1-infected patients, mean age $40.9 \pm 8.1$, of whom 39 females, on adequate diets, all treated applying a therapy plan recommended by international guidelines [2]. One PI and two NRTIs were given to 49 patients: 15 received PI of nelfinavir, 8, lopinavir, 8, indinavir, 5, atazanavir, 5, saquinavir, 4, amprenavir, and 4 , fosamprenavir; all PIs were boosted, with the exception of nelfinavir. One NNRTI and two NRTI were given to 33 patients: 19 received NNRTI of nevirapine and 14, efavirenz. Due to therapeutic regimen failure, the remaining four had to be treated with 1 NRTI +1 NNRTI +1 PI. As NRTI therapy plan, 51 patients received epivir, 35 , stavudine, 30 , didanosine, 28 , zidovudine, 19 , tenofovir, and 5 , abacavir. The third group comprised 30 HIV-1-infected patients, mean age $35.9 \pm 7.9$, of whom 14 females, on adequate diets. None of the group took drugs or nutraceuticals. The treated patients received HAART for an average of $6.3 \pm 2.2$ years. Patients taking at least $80 \%$ of their prescribed medication were arbitrarily considered as having optimal adherence, while those taking under $80 \%$ were considered as having poor adherence. Seventy-one of the $116 \mathrm{HIV}-1$-infected patients were smokers $(22.5 \pm 9.5$ cigarettes daily), of whom 14 in the untreated and 57 in the treated group. There were 20 smokers in the control group (23.1 \pm 9.1 cigarettes daily).

2.2. Oxidative Stress Assessment. Blood samples taken after a minimum of 12 hours' fasting were centrifuged and serum was divided into aliquots and stored at $-80^{\circ} \mathrm{C}$ until used. To assess serum total oxidant and antioxidant levels we utilized the commercially available d-ROMs and OXY-Adsorbent tests (Diacron International, Grosseto, Italy), respectively [21, 22]. d-ROMs serum levels of 250-300 CARR U/mL were considered normal $[21,22]$, and OXY-Adsorbent serum levels higher than $350 \mu$ moles $\mathrm{HClO} / \mathrm{mL}$ were considered normal [22]. These tests were performed according to manufacturers' instructions.

2.3. Other Laboratory Assays and Clinical Data. Glycemia, cholesterolemia, triglyceridemia, alanine aminotransferase (ALT), HBsAg, anti-HBs, anti-HBc, and anti-HCV antibodies were assessed in all enrolled subjects, using the same blood samples used in the oxidative status and antioxidant reef tests. Quantitative serum viral load and blood CD4 T-cells were analyzed in HIV patients. Conventional and ultrasensitive Roche Amplicore HIV Assay (Roche Molecular System, Alameda, CA, USA) was used to assess serum HIV RNA. Viral loads equal or superior to 50 copies $/ \mathrm{mL}$ serum can be detected by this test. CD4 T-cells were assessed by flux cytometry using the IMK multiset commercial kit (Becton Dickinson, Mountain View, CA, USA). Clinical data, including smoking habits, Body Mass Index (BMI), antiretroviral therapy regimen, and HAART adherence, were collected at the time of laboratory tests.

2.4. Statistical Analysis. Data are expressed as mean \pm SD. In preliminary analyses, we used $\chi^{2}$ tests for nominal variables and one-way analysis of variance (ANOVA) (StatisticStatSoft) for continuous variables, to compare data from the three groups analyzed. Statistical significance levels of at least $P<.05$ were used for all tests. To assess the influence of HIVRNA on oxidative status, the mean d-ROMs levels of treated patients with $\leq 400$ copies/mL HIV-RNA were compared to those of treated patients with $>400$ copies $/ \mathrm{mL}$ (ANOVA). The mean d-ROMs levels of treated patients with $\geq 350 / \mathrm{mL}$ CD4 T-cell count and those of treated patients with $<350 / \mathrm{mL}$ CD4 T-cell count were compared (ANOVA), to assess the influence of immunological conditions on oxidative status. To evaluate the influence of normal and altered levels of glycemia, ALT, cholesterolemia, and triglyceridemia on oxidative status, we compared (ANOVA) mean d-ROMs levels in treated patients with glycemia $\leq 100 \mathrm{mg} / \mathrm{dL}$ versus > $100 \mathrm{mg} / \mathrm{dL}, \mathrm{ALT} \leq 41 \mathrm{U} / \mathrm{L} 8$ versus $>41 \mathrm{U} / \mathrm{L}$, cholesterolemia $\leq 180 \mathrm{mg} / \mathrm{dL}$ versus $>180 \mathrm{mg} / \mathrm{dL}$, and triglyceridemia $\leq$ $170 \mathrm{mg} / \mathrm{dL}$ versus $>170 \mathrm{mg} / \mathrm{dL}$. In order to render results more evident, we also determined the Odds Ratio (OR) for the above parameters and multivariate logistic regression for the clinical and laboratory variables of treated patients.

\section{Results}

3.1. Clinical Data. The clinical data of the 116 HIV-1infected patients (86 HAART-treated and 30 untreated) and of 46 controls are given in Table 1. As shown, the three groups differ significantly regarding age, number of smokers, BMI, cholesterolemia, triglyceridemia, and ALT and HIVRNA levels.

3.2. Comparisons of Oxidative Balance Markers in HIV1 Treated and Untreated Groups and Controls. Oxidative balance markers for the three experimental groups are given in Tables 2 and 3. The number of subjects with increased d-ROMs ( $>300 \mathrm{CARR} \mathrm{U} / \mathrm{mL}$ ) was significantly higher in HIV-1 patients compared to controls $(P=.000)$. 
TABLe 1: Clinical data of HIV patients and controls.

\begin{tabular}{|c|c|c|c|c|}
\hline & Controls & HIV-1-treated & HIV-1-untreated & $P$ \\
\hline Number & 46 & 86 & 30 & \\
\hline Age (years) & $39.5 \pm 11.4$ & $40.9 \pm 8.1$ & $35.9 \pm 7.9$ & $.038^{*}$ \\
\hline Age range & 21-61 & $16-65$ & 21-45 & \\
\hline Female/male & $21 / 25$ & $39 / 47$ & $14 / 16$ & $.992^{*}$ \\
\hline Current smokers & 20 & 57 & 14 & $.021^{\S}$ \\
\hline Cigarettes daily & $23.1 \pm 9.1$ & $25.7 \pm 9.8$ & $21.7 \pm 9.4$ & $.290^{*}$ \\
\hline BMI & $21.7 \pm 1.4$ & $22.8 \pm 2.7$ & $23.0 \pm 2.2$ & $.017^{*}$ \\
\hline Cholesterolemia (mg/dL) & $183.0 \pm 27.4$ & $195.4 \pm 39.4$ & $164.7 \pm 25.4$ & $.000^{*}$ \\
\hline Triglyceridemia (mg/dL) & $84.7 \pm 39.8$ & $181.1 \pm 179.8$ & $90.5 \pm 38.9$ & $.000^{*}$ \\
\hline Glycemia (mg/dL) & $88.4 \pm 10.3$ & $89.7 \pm 19.2$ & $90.5 \pm 10.8$ & $.836^{*}$ \\
\hline $\operatorname{ALT}(\mathrm{UI} / \mathrm{L})$ & $21.1 \pm 8.2$ & $46.5 \pm 38.9$ & $40.13 \pm 36.5$ & $.000^{*}$ \\
\hline $\mathrm{HCV}$ positive & 0 & 39 & 13 & \\
\hline HBV positive & 0 & 1 & 0 & \\
\hline $\mathrm{HCV}+\mathrm{HDV}$ positive & 0 & 4 & 1 & \\
\hline HAART-PI & - & 49 & - & \\
\hline HAART-NNRTI & - & 33 & - & \\
\hline HAART-PI + NNRTI & - & 4 & - & \\
\hline CD4 T-cell count (cells/mL) & nd & $554.4 \pm 279.2$ & $528.9 \pm 119.5$ & $.629^{*}$ \\
\hline CD4 T-cell count $\geq 500$ cells $/ \mathrm{mL}$ & - & 46 & 16 & $.864^{\S}$ \\
\hline CD4 T-cell count 499-200 cells $/ \mathrm{mL}$ & - & 32 & 14 & $.693^{\S}$ \\
\hline CD4 T-cell count $<200$ cells/mL & - & 8 & 0 & $.220^{\S}$ \\
\hline HIV-RNA level $\left(\log _{10} \mathrm{cp} / \mathrm{mL}\right)$ & nd & $2.2 \pm 0.8$ & $4.1 \pm 0.4$ & $.000^{*}$ \\
\hline HIV-RNA $<400 \mathrm{cp} / \mathrm{mL}$ & - & 69 & 0 & \\
\hline
\end{tabular}

Data are expressed as mean \pm SD.

* Calculated by One-Way ANOVA.

${ }^{\S}$ Calculated by $\chi^{2}$.

As expected, HIV-1-infected patients display higher serum oxidant levels than controls $(P=.000)$ (Table 2). However, within the HIV-1-infected group, untreated patients had significantly lower oxidant levels than treated ones $(P=.001$, Table 3). Oxidative status was more pronounced in females compared to males in all three groups considered. However, in untreated patients, gender difference was not statistically significant $(P=.08)$ (Table 3$)$.

The number of subjects with decreased OXY-Adsorbent (< $350 \mu \mathrm{mol} \mathrm{HCLO} / \mathrm{mL}$ ) was not statistically different among the three groups (Table 2). Treated patients had antioxidant reef levels significantly lower than controls and untreated patients $(P=.000)$. No significant difference in antioxidant reef levels was observed in males and females treated with HAART $(P=.59)$, while control and untreated males had antioxidant reef levels which were significantly lower than corresponding females $(P=.027, P=.018$, resp.).

3.3. Oxidative Balance and Smoking. Smoking is linked to an increase in oxidative stress $[19,20]$. For this reason we next assessed the influence of smoking on oxidative balance in the three experimental groups.

As shown in Table 2, d-ROMs was significantly higher in treated patients-both smokers and nonsmokerscompared to controls and untreated patients—-both smokers and nonsmokers $(P=.000)$. On the other hand, OXYAdsorbent was lower in treated smokers compared to those in the control and untreated groups $(P=.000)$, while no difference in OXY-Adsorbent was observed in nonsmokers in the three groups (Table 2). As expected, control smokers had higher d-ROMs levels than control nonsmokers $(P=.001)$. However, smoking significantly affected d-ROMs in neither treated nor untreated patients $(P=.8, P=.2$, resp. $)$.

OXY-Adsorbent levels did not differ in smoker and nonsmoker controls $(P=.299)$ nor in smoker and nonsmokeruntreated patients $(P=.841)$, while they were significantly higher in nonsmoker-treated patients compared to smoker ones $(P=.022)$.

3.4. Oxidative Status and HAART. Given the high d-ROMs levels in treated patients, it seemed to us to be of interest to evaluate the relationship between oxidative status and HAART adherence. Patients with optimal adherence had significantly higher d-ROMs levels than those with poor adherence $(P=.008$; OR $=4.2,95 \%$ C.I. of $1.1-15.5)$ (Table 3). Differences in the following variables CD4 T-cell count, HIV-RNA level, antiretroviral-drug class, HCV coinfection, ALT, glycemia, cholesterolemia, and triglyceridemia were not significantly linked to d-ROMs levels (Table 3). An additional increase in d-ROMs was observed in patients receiving HAART plus psychiatric drugs (Table 3). 
TABLE 2: Comparison of d-ROMs and Oxy-Adsorbent tests between the three groups.

\begin{tabular}{|c|c|c|c|c|}
\hline & Controls & HIV-1 treated & HIV-1 untreated & $P$ \\
\hline d-ROMs (CARR U/mL) & $295.4 \pm 58.9$ & $366.1 \pm 68.8$ & $322.1 \pm 41.2$ & $.000^{*}$ \\
\hline d-ROMs (CARR U/mL) in females & $319.7 \pm 66.6$ & $393.0 \pm 78.4$ & $336.3 \pm 49.1$ & $.000^{*}$ \\
\hline d-ROMs (CARR U/mL) in males & $275.0 \pm 43.1$ & $343.8 \pm 50.5$ & $309.6 \pm 29.0$ & $.000^{*}$ \\
\hline d-ROMs increased: no. of subjects & 18 & 72 & 18 & $.000^{\S}$ \\
\hline OXY-Adsorbent ( $\mu \mathrm{mol} \mathrm{HClO} / \mathrm{mL})$ & $433.6 \pm 73$ & $411.6 \pm 48.2$ & $458.7 \pm 50.6$ & $.000^{*}$ \\
\hline OXY-Adsorbent $(\mu \mathrm{mol} \mathrm{HClO} / \mathrm{mL})$ in females & $461.1 \pm 77.6$ & $408.5 \pm 44.3$ & $477.2 \pm 57.1$ & $.000^{*}$ \\
\hline Oxy-Adsorbent $(\mu \mathrm{mol} \mathrm{HClO} / \mathrm{mL})$ in males & $410.6 \pm 61.4$ & $414.1 \pm 51.4$ & $430.0 \pm 53.5$ & $.228^{*}$ \\
\hline Oxy-Adsorbent reduced: no. of subjects & 4 & 6 & 0 & $.276^{\S}$ \\
\hline \multicolumn{5}{|l|}{ Smokers } \\
\hline d-ROMs (CARR U/mL) & $325.9 \pm 68.8$ & $362.1 \pm 60.4$ & $312.1 \pm 30.0$ & $.000^{*}$ \\
\hline Oxy-Adsorbent ( $\mu \mathrm{mol} \mathrm{HClO} / \mathrm{mL})$ & $420.7 \pm 67.3$ & $403.2 \pm 45.0$ & $457.6 \pm 59.1$ & $.000^{*}$ \\
\hline \multicolumn{5}{|l|}{ Nonsmokers } \\
\hline d-ROMs (CARR U/mL) & $272.0 \pm 36.6$ & $366.1 \pm 56.4$ & $331.4 \pm 48.7$ & $.000^{*}$ \\
\hline Oxy-Adsorbent $(\mu \mathrm{mol} \mathrm{HClO} / \mathrm{mL})$ & $443.5 \pm 76.9$ & $428.1 \pm 50.6$ & $453.1 \pm 61.8$ & $.113^{*}$ \\
\hline
\end{tabular}

Data are expressed as mean $\pm \mathrm{SD}$.

* Calculated by One-Way ANOVA.

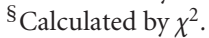

Multivariate logistic regression confirmed significant oxidative-status increase in optimal-adherence-treated patients $(P=.032)$ and in women $(P=.005)$, while they did not reveal any link between HAART and psychiatric drugs $(P=.14)$.

\section{Discussion}

The d-ROMs and OXY-Adsorbent tests, both available commercially, were used to determine oxidative status and antioxidant-reef status, respectively, in the serum of one treated and one untreated group of HIV-1-infected patients and of one control group. It has been widely reported that HIV infection causes oxidative stress [8, 23-25], which is an imbalance between oxidant and antioxidant status. Under our experimental conditions, significantly higher d-ROMs levels were found in HIV-1-infected patientsboth treated and untreated-compared to controls, while OXY-Adsorbent levels were significantly reduced in HAART patients. However, as reported by other authors [26], we too found that treated HIV-1-infected patients had significantly higher d-ROMs levels than untreated ones or controls. Taken together, our results indicate that HAART may affect oxidative stress in HIV-1-infected patients and suggest that antiretroviral therapy plays an important role in the synergy of HIV infection and oxidative stress [26]. Accordingly, under our experimental conditions, when treated patients alone are considered, viral load does not seem to affect oxidative status. Very significantly, patients who rigorously follow antiretroviral therapy (optimal HAART adherence) have higher oxidative status than those who do not closely follow the therapy (poor HAART adherence). Different therapy regimens do not seem to affect oxidative status. Analysis of variance revealed no significant further increase in oxidative status in HIV-1-infected patients taking antiretroviral and other drugs (antibiotics, antihypertensive drugs, painkillers, lipid-lowering agents), with the exception of psychiatric drugs (e.g., anxiolytics or antidepressants). However, multivariate logistic regression analysis confirmed the statistical significance of these findings for only two variables: optimal HAART adherence and female gender. CD4 T-cell count, HIV-HCV coinfection, glycemia, cholesterolemia, and triglyceridemia did not appear to correlate with oxidative status. However, triglyceridemia and cholesterolemia were significantly lower in controls and untreated patients compared to treated ones, highlighting the fact that HAART causes dislipidemia [27]. This condition, in turn, may increase the risk of cardiovascular disorders. The antioxidant component, assessed (with the OXY-Adsorbent test) as serum total antioxidant capacity, was within normal levels ( $>350 \mu \mathrm{mol} \mathrm{HClO} / \mathrm{mL}$ ) in most HIV-1 patients enrolled. This finding is only apparently in contrast with literature [17], which reports reduced antioxidant capacity in HIV-infected patients, predominantly those at an advanced stage of the illness. In fact, the pathology status of HIV-1-infected patients under study, except for 8 treated patients (6.9\%) with less than 200 CD4 T-cell/mL, was not yet advanced. It is of interest that the $30 \mathrm{HIV}$-1-infected patients who, given their adequate immunological and virological states, were untreated, had a higher antioxidant reef than HAART-treated patients. The lowest antioxidant-reef levels were found in treated patients, while the highest ones were found in untreated patients. These findings are not readily explainable; however, the lowest antioxidant-reef levels - which were found in treated patients-might be not only due to the therapy itself but also due to an increased utilization of endogenous antioxidants, following increased ROS production in these patients. The remaining important aspects of our study concern gender and smoking. Oxidative status in female-treated patients and female controls was significantly higher than in males. A possible explanation is 
TABLE 3: Comparison of d-ROMs concentration in the three groups, dichotomically classified by clinical and laboratory variables.

\begin{tabular}{|c|c|c|c|c|}
\hline \multirow{2}{*}{$\begin{array}{l}\text { Variables } \\
\text { Controls }\end{array}$} & \multicolumn{2}{|c|}{ d-ROMs } & \multirow[t]{2}{*}{$P^{*}$} & \multirow[t]{2}{*}{ OR (CI 95\%) } \\
\hline & & & & \\
\hline \multirow{2}{*}{ Gender } & Female & Male & & \\
\hline & $319.7 \pm 66.6$ & $275.0 \pm 43.1$ & .009 & $6.5(1.7-24.3)$ \\
\hline \multirow{2}{*}{ Smoking } & Yes & No & & \\
\hline & $325.9 \pm 68.8$ & $272.0 \pm 36.6$ & .001 & $6.3(1.7-23.7)$ \\
\hline \multicolumn{5}{|l|}{ HIV-1 infection } \\
\hline \multirow{2}{*}{ HAART } & Yes & No & & \\
\hline & $366.1 \pm 68.6$ & $322.4 \pm 41.5$ & .001 & $3.4(1.3-8.7)$ \\
\hline \multicolumn{5}{|l|}{ HIV-1 treated } \\
\hline \multirow{2}{*}{ Gender } & Female & Male & & \\
\hline & $393.0 \pm 78.4$ & $343.8 \pm 50.5$ & .000 & $3.7(0.9-14.2)$ \\
\hline \multirow{2}{*}{ Smoking } & Yes & No & & \\
\hline & $362.1 \pm 60.4$ & $366.1 \pm 56.4$ & .8 & $0.7(0.2-2.6)$ \\
\hline CD4 T-cells & $\begin{array}{c}\geq 350 \text { cells } / \mathrm{mL} \\
364.0 \pm 69.1\end{array}$ & $\begin{array}{c}<350 \text { cells } / \mathrm{mL} \\
373.1 \pm 69.0\end{array}$ & .6 & $0.9(0.2-3.5)$ \\
\hline \multirow{2}{*}{ HIV-RNA } & $\leq 400 \mathrm{cp} / \mathrm{mL}$ & $>400 \mathrm{cp} / \mathrm{mL}$ & .7 & $1.1(0.3-4.6)$ \\
\hline & $364.6 \pm 70.1$ & $372.3 \pm 64.8$ & & \\
\hline \multirow{2}{*}{ Drug class } & PI & NNRTI & & \\
\hline & $372.5 \pm 75.1$ & $359.7 \pm 61.6$ & .4 & $1.3(0.4-4.4)$ \\
\hline \multirow{2}{*}{ Nelfinavir } & Yes & No & & \\
\hline & $398.2 \pm 95.6$ & $359.9 \pm 61.2$ & .056 & $\infty$ \\
\hline \multirow{2}{*}{ Nevirapine } & Yes & No & & \\
\hline & $372.3 \pm 63.4$ & $364.4 \pm 70.6$ & .7 & $1.8(0.4-9.1)$ \\
\hline \multirow{2}{*}{ Efavirenz } & Yes & No & & \\
\hline & $348.1 \pm 54.2$ & $370.6 \pm 71.6$ & .2 & $0.4(0.1-1.5)$ \\
\hline \multirow{2}{*}{ HAART adherence } & Optimal & Poor & & \\
\hline & $378.5 \pm 71.7$ & $336.0 \pm 50.7$ & .008 & $4.2(1.1-15.5)$ \\
\hline \multirow{2}{*}{ Other drugs + HAART } & Yes & No & & \\
\hline & $371.8 \pm 61.7$ & $363.1 \pm 72.7$ & .6 & $2.2(0.6-8.6)$ \\
\hline \multirow{2}{*}{ Psychiatric drugs + HAART } & Yes & No & & \\
\hline & $399.4 \pm 51.5$ & $359.7 \pm 70.1$ & .047 & $\infty$ \\
\hline \multirow{2}{*}{ HIV-HCV co-infected } & Yes & No & & \\
\hline & $359.7 \pm 57.9$ & $371.3 \pm 79.1$ & .5 & $1.180 .3-3.3$ \\
\hline ALT & $\begin{array}{c}\leq 41 \mathrm{UI} / \mathrm{L} \\
374.2 \pm 80.8\end{array}$ & $\begin{array}{c}<40 \mathrm{UI} / \mathrm{L} \\
355.0 \pm 46.3\end{array}$ & .2 & $1.0(0.33-3.3)$ \\
\hline Glycemia & $\begin{array}{l}\leq 100 \mathrm{mg} / \mathrm{dL} \\
372.3 \pm 71.1\end{array}$ & $\begin{array}{l}<100 \mathrm{mg} / \mathrm{dL} \\
342.9 \pm 54.9\end{array}$ & .1 & $2.3(0.7-8.1)$ \\
\hline Cholesterolemia & $\begin{array}{l}\leq 180 \mathrm{mg} / \mathrm{dL} \\
355.0 \pm 61.6\end{array}$ & $\begin{array}{l}>180 \mathrm{mg} / \mathrm{dL} \\
372.7 \pm 72.5\end{array}$ & .2 & $0.7(0.2-2.4)$ \\
\hline Triglyceridemia & $\begin{array}{c}\leq 170 \mathrm{mg} / \mathrm{dL} \\
371.2 \pm 79.4\end{array}$ & $\begin{array}{c}>170 \mathrm{mg} / \mathrm{dL} \\
358.4 \pm 48.5\end{array}$ & .4 & $0.8(0.2-2.8)$ \\
\hline HIV-1 untreated & & & & \\
\hline & Female & Male & & \\
\hline Gender & $336.3 \pm 49.1$ & $309.6 \pm 28.9$ & .076 & $0.8(0.2-3.5)$ \\
\hline Smoking & Yes & No & & \\
\hline Smoking & $312.1 \pm 30.0$ & $331.4 \pm 48.7$ & .2 & $0.8(0.2-3.5)$ \\
\hline HIV-HCV co-infected & Yes & No & & \\
\hline & $330.4 \pm 38.9$ & $314.7 \pm 43.0$ & .3 & $2.5(0.5-11.4)$ \\
\hline ALT & $\begin{array}{c}\leq 41 \mathrm{UI} / \mathrm{L} \\
316.9 \pm 40.2\end{array}$ & $\begin{array}{c}<40 \mathrm{UI} / \mathrm{L} \\
332.4 \pm 43.3\end{array}$ & .3 & $0.2(0.04-1.4)$ \\
\hline
\end{tabular}

Data are expressed as mean $\pm \mathrm{SD}$.

${ }^{*}$ Calculated by One-Way ANOVA. 
that in these two groups there were several women of perimenopausal or menopausal age, whose conditions, as is known, increase ROS production [28, 29]. By contrast, the majority of untreated female patients under study were of fertile age and did not significantly differ in oxidative status from males in the same group. Our study, as did previous ones $[19,20]$, revealed (as expected) that smoker controls had a significant increase in oxidant levels compared to nonsmoker controls. Surprisingly, no difference in oxidative status between treated and untreated HIV1 -infected groups was seen when, within these groups, smokers and nonsmokers were compared, even though the number of smokers in the HIV-1 group was significantly higher than in the control group. This result suggests that increased oxidative stress in HIV-1 patients mainly depends on the infection and/or antiretroviral therapy.

\section{Conclusion}

In summary, our results show that HIV-1 infection increases oxidative status, and that it is further increased by HAART. Consequently they suggest that HAART plays an important role in determining oxidative stress in these patients. Supporting this, patients with optimal adherence show higher oxidative stress than those with poor adherence. In addition, they suggest that antiretroviral therapy also affects metabolic status. Viral load and CD4 do not appear to be involved in oxidative stress occurring in HIV-1 patients.

\section{Competing Interests}

The authors declare that they have no competing and/or financial interests.

\section{Authors' Contributions}

AM was primarily responsible for the design of the study, for developing the analysis concept, for analysis of data, and for writing the manuscript. ELI provided help with oxidative balance determination. MGC and $\mathrm{CB}$ were responsible for data management. AM and DC were responsible for analysis. SD oversaw all aspects of this study. NC was responsible for the biochemical parts and participated in the writing of the study. All authors have read and approved the final manuscript.

\section{Acknowledgment}

The authors wish to thank volunteers and HIV-1 patients, all of whom enthusiastically agreed to participate in this study. They would also like to thank Professor Angelo Balestrieri for his invaluable general advice and Professor Edward John Steeden for his excellent help in translating this paper. The technical assistance given by Noemi Ruggeri is much appreciated.

\section{References}

[1] K. Porter, "Survival after introduction of HAART in people with known duration of HIV-1 infection," The Lancet, vol. 355, no. 9210, pp. 1158-1159, 2000.

[2] "Guidelines for the use of antiretroviral agents in HIV-1infected adults and adolescents," Developed by the panel on clinical practices for treatment of HIV infection convened by Department of Health and Human Service (DHHS), http://AIDSinfo.nih.gov.

[3] S. Grabar, V. Le Moing, C. Goujard, et al., "Clinical outcome of patients with HIV-1 infection according to immunologic and virologic response after 6 months of highly active antiretroviral therapy," Annals of Internal Medicine, vol. 133, no. 6, pp. 401-410, 2000.

[4] S. D. Fisher, T. L. Miller, and S. E. Lipshultz, "Impact of HIV and highly active antiretroviral therapy on leucocyte adhesion molecules, arterial inflammation, dyslipidemia, and atherosclerosis," Atherosclerosis, vol. 185, pp. 1-11, 2006.

[5] C. Hadigan, "Diabetes, insulin resistance, and HIV," Current Infectious Disease Reports, vol. 8, no. 1, pp. 69-75, 2006.

[6] S. C. J. M. Vervoort, J. C. C. Borleffs, A. I. M. Hoepelman, and M. H. F. Grypdonck, "Adherence in antiretroviral therapy: a review of qualitative studies," AIDS, vol. 21, no. 3, pp. 271281, 2007.

[7] B. Halliwell, "Free radicals, antioxidants, and human disease: curiosity, cause, or consequence?" The Lancet, vol. 344, no. 8924, pp. 721-724, 1994.

[8] R. Schreck, P. Rieber, and P. A. Baeuerle, "Reactive oxygen intermediates as apparently widely used messengers in the activation of the NF- $\kappa \mathrm{B}$ transcription factor and HIV-1," The EMBO Journal, vol. 10, no. 8, pp. 2247-2258, 1991.

[9] I. I. Kruman, A. Nath, and M. P. Mattson, "HIV-1 protein tat induces apoptosis of hippocampal neurons by a mechanism involving caspase activation, calcium overload, and oxidative stress," Experimental Neurology, vol. 154, no. 2, pp. 276-288, 1998.

[10] J. A. Martín, J. Sastre, J. De la Asunción, F. V. Pallardó, and J. Vinña, "Hepatic $\gamma$-cystathionase deficiency in patients with AIDS," Journal of the American Medical Association, vol. 285, no. 11, pp. 1444-1445, 2001.

[11] T. Hulgan, J. Morrow, R. T. D’Aquila, et al., "Oxidant stress is increased during treatment of human immunodeficiency virus infection," Clinical Infectious Diseases, vol. 37, no. 12, pp. 1711-1717, 2003.

[12] B. J. Day and W. Lewis, "Oxidative stress in NRTI-induced toxicity: evidence from clinical experience and experiments in vitro and in vivo," Cardiovascular Toxicology, vol. 4, no. 3, pp. 207-216, 2004.

[13] G. N. Kumar, J. Dykstra, E. M. Roberts, et al., "Potent inhibition of the cytochrome P-450 3A-mediated human liver microsomal metabolism of a novel HIV protease inhibitor by ritonavir: a positive drug-drug interaction," Drug Metabolism and Disposition, vol. 27, no. 8, pp. 902-908, 1999.

[14] W. Lewis, "Mitochondrial dysfunction and nucleoside reverse transcriptase inhibitor therapy: experimental clarifications and persistent clinical questions," Antiviral Research, vol. 58, no. 3, pp. 189-197, 2003.

[15] A. Cossarizza and G. Moyle, "Antiretroviral nucleoside and nucleotide analogues and mitochondria," AIDS, vol. 18, no. 2 , pp. 137-151, 2004.

[16] J. de La Asunción, M. L. del Olmo, J. Sastre, et al., "AZT treatment induces molecular and ultrastructural oxidative 
damage to muscle mitochondria. Prevention by antioxidant vitamins," The Journal of Clinical Investigation, vol. 102, no. 1, pp. 4-9, 1998.

[17] P. K. Drain, R. Kupka, F. Mugusi, and W. W. Fawzi, "Micronutrients in HIV-positive persons receiving highly active antiretroviral therapy," The American Journal of Clinical Nutrition, vol. 85, no. 2, pp. 333-345, 2007.

[18] A. M. Tang, J. Lanzillotti, K. Hendricks, et al., "Micronutrients: current issues for HIV care providers," AIDS, vol. 19, no. 9, pp. 847-861, 2005.

[19] P. A. Kirkham, G. Spooner, C. Ffoulkes-Jones, and R. Calvez, "Cigarette smoke triggers macrophage adhesion and activation: role of lipid peroxidation products and scavenger receptor," Free Radical Biology and Medicine, vol. 35, no. 7, pp. 697-710, 2003.

[20] J. Helmersson, A. Larsson, B. Vessby, and S. Basu, "Active smoking and a history of smoking are associated with enhanced prostaglandin F2 $\alpha$, interleukin- 6 and F2isoprostane formation in elderly men," Atherosclerosis, vol. 181, no. 1, pp. 201-207, 2005.

[21] K. Dohi, K. Satoh, T. Nakamachi, et al., "Does Edaravone (MCI-186) act as an antioxidant and a neuroproctetor in experimental traumatic brain injury?" Antioxid Redox Signal, vol. 9, pp. 281-287, 2007.

[22] R. Trotti, M. Carratelli, M. Barbieri, et al., "Oxidative stress and a thrombophilic condition alcoholics without severe liver disease," Haematologica, vol. 86, no. 1, pp. 85-91, 2001.

[23] G. W. Pace and C. D. Leaf, "The role of oxidative stress in HIV disease," Free Radical Biology and Medicine, vol. 19, no. 4, pp. 523-528, 1995.

[24] T. S. Dobmeyer, S. Findhammer, J. M. Dobmeyer, et al., "Ex vivo induction of apoptosis in lymphocytes is mediated by oxidative stress: role for lymphocyte loss in HIV infection," Free Radical Biology and Medicine, vol. 22, no. 5, pp. 775-785, 1997.

[25] L. Gil, G. Martínez, I. González, et al., "Contribution to characterization of oxidative stress in HIV/AIDS patients," Pharmacological Research, vol. 47, no. 3, pp. 217-224, 2003.

[26] J. L. Ngondi, J. Oben, D. M. Forkah, L. H. Etame, and D. Mbanya, "The effect of different combination therapies on oxidative stress markers in HIV infected patients in Cameroon," AIDS Research and Therapy, vol. 3, no. 1, p. 19, 2006.

[27] J. A. Aberg, "Lipid management in patients who have HIV and are receiving HIV therapy," Endocrinology and Metabolism Clinics of North America, vol. 38, no. 1, pp. 207-222, 2009.

[28] S. Maffei, A. Mercuri, C. Prontera, G. C. Zucchelli, and C. Vassalle, "Vasoactive biomarkers and oxidative stress in healthy recently postmenopausal women treated with hormone replacement therapy," Climacteric, vol. 9, no. 6, pp. 452458, 2006.

[29] T. Ide, H. Tsutsui, N. Ohashi, et al., "Greater oxidative stress in healthy young men compared with premenopausal women," Arteriosclerosis, Thrombosis, and Vascular Biology, vol. 22, no. 3, pp. 438-442, 2002. 

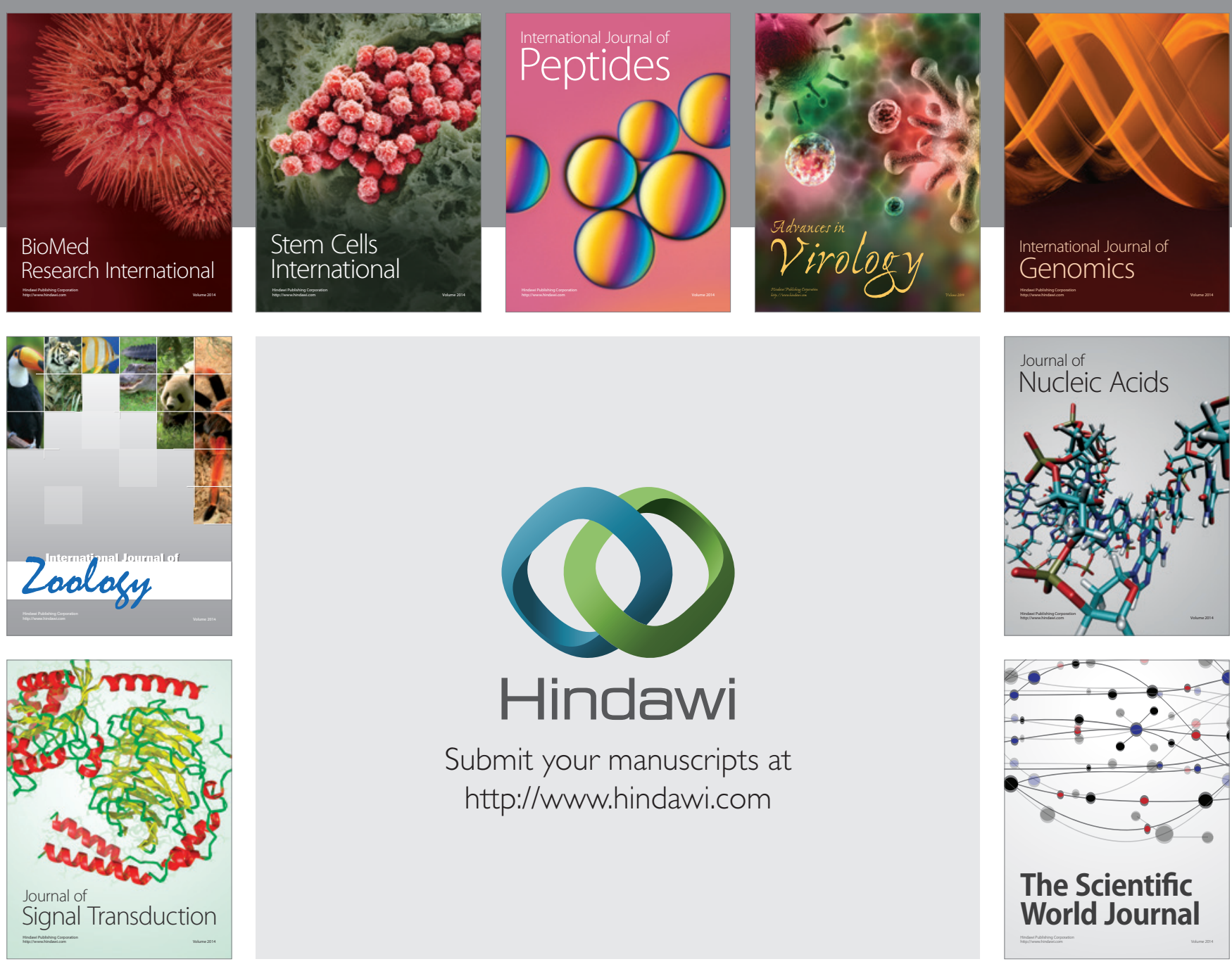

Submit your manuscripts at

http://www.hindawi.com
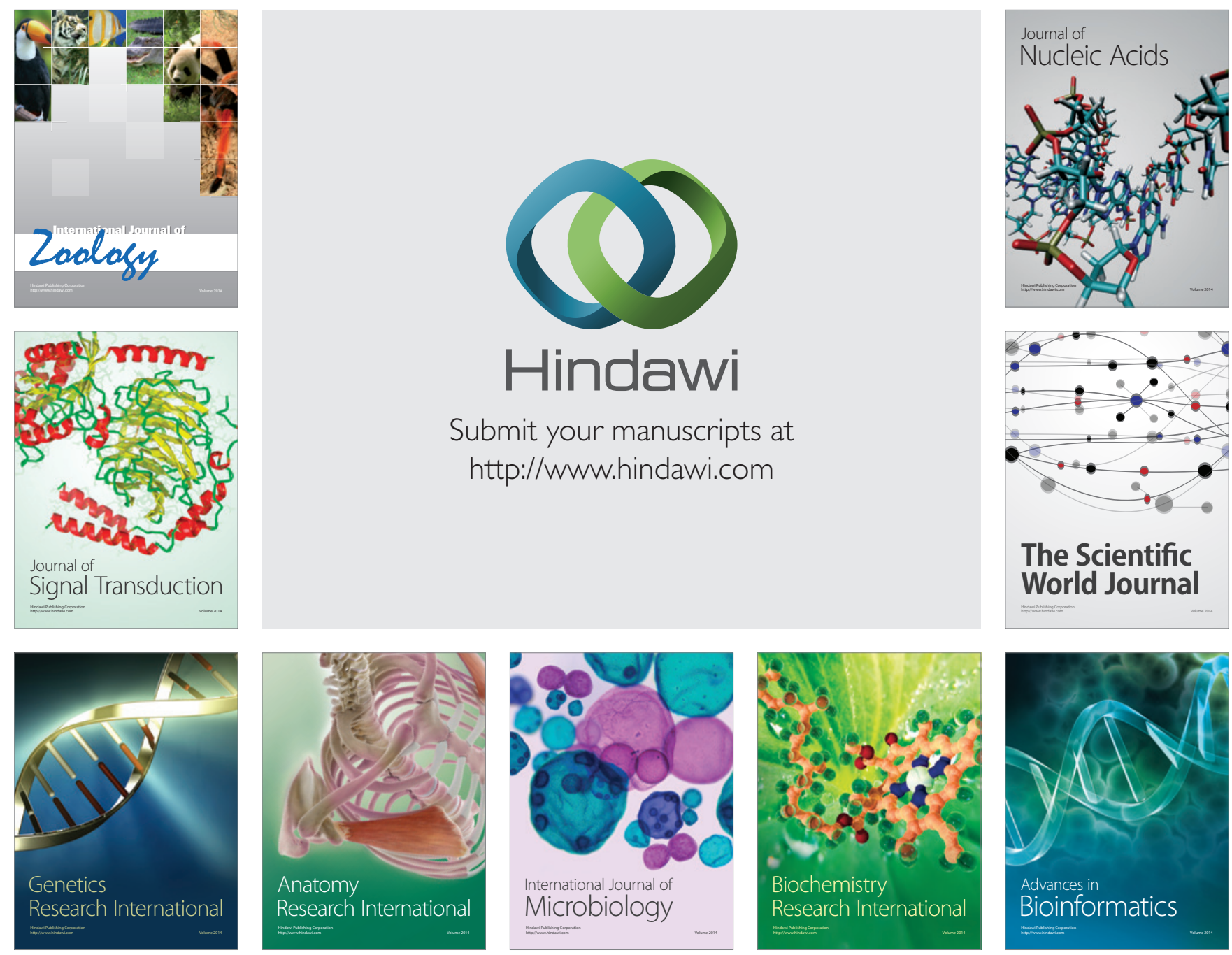

The Scientific World Journal
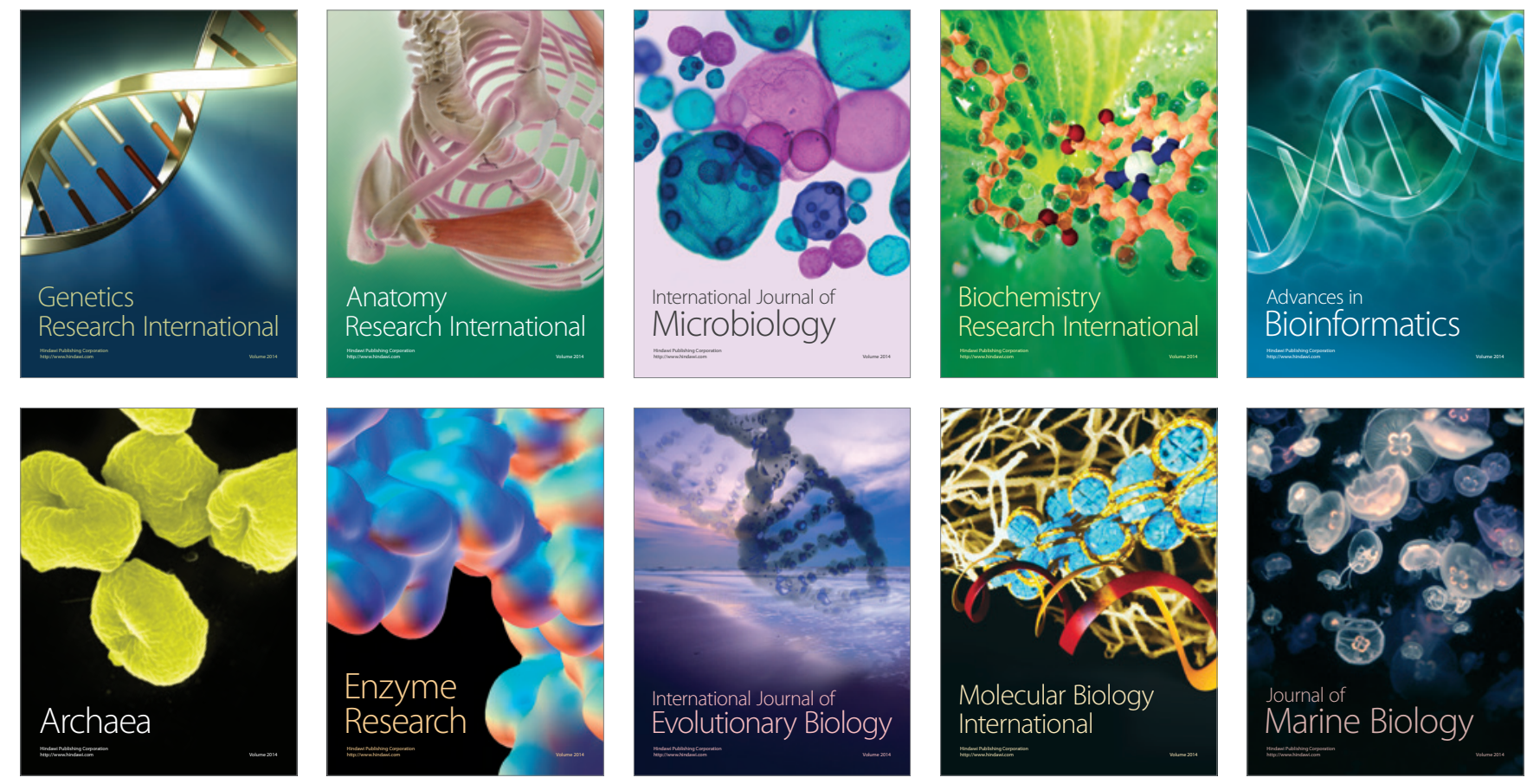